\title{
Two-Stage Stratified Randomized Response Model with Fuzzy Numbers
}

\author{
Mohammad Faisal Khan, ${ }^{1}$ Neha Gupta, ${ }^{2}$ and Irfan $\mathrm{Ali}^{2}$ \\ ${ }^{1}$ College of Science and Theoretical Studies, Saudi Electronic University, P.O. Box 93499, Riyadh 11673, Saudi Arabia \\ ${ }^{2}$ Department of Statistics and Operations Research, Aligarh Muslim University, Aligarh 202002, India \\ Correspondence should be addressed to Mohammad Faisal Khan; faisalkhan004@yahoo.com
}

Received 16 November 2015; Revised 3 February 2016; Accepted 4 February 2016

Academic Editor: Ashok B. Kulkarni

Copyright (c) 2016 Mohammad Faisal Khan et al. This is an open access article distributed under the Creative Commons Attribution License, which permits unrestricted use, distribution, and reproduction in any medium, provided the original work is properly cited.

\begin{abstract}
We consider an allocation problem in two-stage stratified Warner's randomized response model and minimize the variance subject to cost constraint. The costs (measurement costs and total budget of the survey) in the cost constraint are assumed as fuzzy numbers, in particular triangular and trapezoidal fuzzy numbers due to the ease of use. The problem formulated is solved by using Lagrange multipliers technique and the optimum allocation obtained in the form of fuzzy numbers is converted into crisp form using $\alpha$-cut method at a prescribed value of $\alpha$. An illustrative numerical example is presented to demonstrate the proposed problem.
\end{abstract}

\section{Introduction}

Sample survey is a method of drawing an inference about the characteristic of a population or universe by observing only a part of the population. In modern complex surveys it is not possible to obtain true measurements on all the characteristics of interest on all the units in the sample because they are affected by two types of errors, that is, sampling errors and nonsampling errors. Nonsampling error is further classified into two types, response and nonresponse errors. Reduction in the reliability of measurements results in response error which can be minimized over repeated measurements. Whereas nonresponse errors are due to the nonavailability of information about some selected units for one or the other reason.

A major source of nonresponse errors in sample surveys is the difficulty to obtain true responses when respondents are asked questions of highly personal or controversial nature, for example, questions on accumulated savings, intentional tax evasion, consumption of illegal drugs, and extramarital affairs. To avoid providing the requisite information or to avoid embarrassment, some respondents may refuse to answer or may intentionally give wrong answers. Thus the estimates obtained from a direct survey on such topics would be subject to high bias and any inference drawn from these would be erroneous. In order to solve this problem Warner [1] introduced a randomized response technique (RRT) which was developed subsequently by different authors.

Some other authors who introduce other randomized response techniques are Mangat and Singh [2], Chua and Tsui [3], Padmawar and Vijayan [4], Chang and Huang [5], Chaudhuri [6], and so forth. Kim and Warde [7] suggested a stratified randomized response using optimum allocation. Mangat and Singh [2] proposed a two-stage randomized response model.

Traditional decision making problems are handled either by the deterministic approach or by probabilistic approach. Deterministic approach completely avoiding the uncertainty provides an approximate solution while probabilistic approach on an assumption represents any uncertainty as a probability distribution. Both of these approaches only partially capture reality. Uncertainty also is involved in decision problems due to vagueness or impreciseness associated with linguistic information; then in this case optimization using fuzzy mathematical theories becomes more relevant. The idea of fuzzy decision making problems was proposed by Bellmann and Zadeh [8] and this idea was used in problems of mathematical programming by Tanaka and Asai [9]. Many authors use fuzzy data/fuzzy numbers in decision making problems such as Mahapatra and Roy [10], Pramanik and 
Roy [11], Abbasbandy and Hajjari [12], Kaur and Kumar [13], Ebrahimnejad [14], Sen et al. [15], and Gupta and Bari [16].

In this paper, the deterministic problem formulated by Ghufran et al. [17] is extended by considering it into an uncertain environment. Here we consider the measurement cost and total budget for the survey as fuzzy numbers and formulate a fuzzy nonlinear programming problem. Then, the fuzzy nonlinear problem is solved by Lagrange multipliers technique after converting it into crisp problem using $\alpha$ cut. For demonstrating the proposed problem an illustrative example is presented.

\section{Preliminary}

Before formulating the problem of interest, we should know the basic definitions of fuzzy sets, fuzzy numbers, and so forth, which are reproduced here, from Bector and Chandra [18], Mahapatra and Roy [10], Hassanzadeh et al. [19], and Aggarwal and Sharma [20] as follows.

Fuzzy Set. A fuzzy set $\widetilde{A}$ in a universe of discourse $X$ is defined as the following set of pairs $\widetilde{A}=\left\{\left(x, \mu_{\widetilde{A}}(x)\right): x \in X\right\}$. Here $\mu_{\widetilde{A}}: X \rightarrow[0,1]$ is a mapping called the membership function of the fuzzy set $\widetilde{A}$ and $\mu_{\widetilde{A}}$ is called the membership value or degree of membership of $x \in X$ in the fuzzy set $\widetilde{A}$. The larger the value of $\mu_{\widetilde{A}}$, the stronger the grade of membership in $\widetilde{A}$.

$\alpha$-Cut. The $\alpha$-cut for a fuzzy set $\widetilde{A}$ is shown by $\widetilde{A}_{\alpha}$ and for $\alpha \in[0,1]$ is defined to be

$$
\widetilde{A}_{\alpha}=\left\{x \mid \mu_{\widetilde{A}}(x) \geq \alpha, x \in X\right\}
$$

where $X$ is the universal set.

Upper and lower bounds for any $\alpha$-cut $\widetilde{A}_{\alpha}$ are given by $\widetilde{A}_{\alpha}^{U}$ and $\widetilde{A}_{\alpha}^{L}$, respectively.

Fuzzy Number. A fuzzy set $A$ in $\mathbb{R}$ is called a fuzzy number if it satisfies the following conditions:

(i) $A$ is convex and normal.

(ii) $A_{\alpha}$ is a closed interval for every $\alpha \in(0,1]$.

(iii) The support of $A$ is bounded.

Triangular Fuzzy Number (TFN). A fuzzy number $\widetilde{A}=$ $(p, q, r)$ is said to be a triangular fuzzy number if its membership function is given by

$$
\mu_{\widetilde{A}}= \begin{cases}\frac{x-p}{q-p}, & \text { if } p \leq x \leq q \\ \frac{r-x}{r-q}, & \text { if } q \leq x \leq r \\ 0, & \text { otherwise. }\end{cases}
$$

Trapezoidal Fuzzy Number (TrFN). A fuzzy set $\widetilde{A}=(p, q, r, s)$ on real numbers $\mathbb{R}$ is called a trapezoidal fuzzy number with membership function as follows:

$$
\mu_{\widetilde{A}}(x)= \begin{cases}0, & x \leq p, \\ \frac{x-p}{q-p}, & p \leq x \leq q, \\ 1, & q \leq x \leq r, \\ \frac{s-x}{s-r}, & r \leq x \leq s, \\ 0, & s \leq x .\end{cases}
$$

\section{Statement of the Problem of Two-Stage Randomized Response Model}

Consider a stratified population of size $N$ partitioned into $L$ disjoint strata of size $N_{h} ; h=1,2, \ldots, L$ and $N=\sum_{h=1}^{L} N_{h}$. Let $W_{h}=N_{h} / N$ denote stratum weights, $n_{h}$ denotes sample size, and $n=\sum_{h=1}^{L} n_{h}$ is the total sample size for the stratum $h$.

In the first stage an individual respondent in the sample is instructed to use the randomization device $R_{1 h}$ which consists of the following two statements.

(i) "I belong to the sensitive group" and (ii) "Go to the randomization device $R_{2 h}$ in the second stage" with known probabilities $M_{h}$ and $\left(1-M_{h}\right)$, respectively.

In the second stage the respondents are instructed to use the randomization device $R_{2 h}$ which consists of the following two statements.

(i) "I belong to the sensitive group" and (ii) "I do not belong to the sensitive group" with known probabilities $P_{h}$ and $\left(1-P_{h}\right)$, respectively.

Assuming that the "Yes" or "No" reports are made truthfully for different outcomes and $M_{h}$ and $P_{h}$ are set by the interviewer, then the probability of a "Yes" answer in stratum $h$ is given by

$$
\begin{array}{r}
Y_{h}=M_{h} \pi_{s h}+\left(1-M_{h}\right)\left[P_{h} \pi_{s h}+\left(1-P_{h}\right)\left(1-\pi_{s h}\right)\right] ; \\
h=1,2, \ldots, L
\end{array}
$$

and $\pi_{s h}$ is the proportion of respondents belonging to the sensitive group from stratum $h$. The maximum likelihood estimate of $\pi_{s h}$ is

$$
\widehat{\pi}_{s h}=\frac{\widehat{Y}_{h}-\left(1-M_{h}\right)\left(1-P_{h}\right)}{2 P_{h}-1+2 M_{h}\left(1-P_{h}\right)} ; \quad h=1,2, \ldots, L,
$$

where $\widehat{Y}_{h}$ is the estimated proportion of "Yes" answers which follows a binomial distribution $B\left(n_{h}, Y_{h}\right)$. It can be seen that the estimator $\widehat{\pi}_{s h}$ is unbiased for $\pi_{s h}$ with variance

$$
\begin{aligned}
V\left(\hat{\pi}_{s h}\right) & \\
= & \frac{\pi_{s h}\left(1-\pi_{s h}\right)}{n_{h}} \\
& +\frac{\left(1-M_{h}\right)\left(1-P_{h}\right)\left[1-\left(1-M_{h}\right)\left(1-P_{h}\right)\right]}{n_{h}\left[2 P_{h}-1+2 M_{h}\left(1-P_{h}\right)\right]^{2}} .
\end{aligned}
$$


If the suffix " $h$ " is removed then the expressions 1,2 , and 3 will be reduced in Mangat and Singh's expressions.

Since $n_{h}$ are drawn independently from each stratum, the estimators for individual strata can be added to obtain the estimator for the whole population. Thus an unbiased estimate of $\pi_{s h}$ is given by

$$
\widehat{\pi}_{s}=\sum_{h=1}^{L} W_{h} \widehat{\pi}_{s h} .
$$

Using (5)

$$
\hat{\pi}_{s}=\sum_{h=1}^{L} W_{h}\left[\frac{\widehat{Y}_{h}-\left(1-M_{h}\right)\left(1-P_{h}\right)}{2 P_{h}-1+2 M_{h}\left(1-P_{h}\right)}\right]
$$

with a sampling variance

$$
V\left(\widehat{\pi}_{s}\right)=\sum_{h=1}^{L} W_{h}^{2} V\left(\widehat{\pi}_{s h}\right)
$$

or

$$
\begin{aligned}
& V\left(\hat{\pi}_{s}\right) \\
& =\sum_{h=1}^{L} \frac{W_{h}^{2}}{n_{h}} \pi_{s h}\left(1-\pi_{s h}\right) \\
& \quad+\frac{\left(1-M_{h}\right)\left(1-P_{h}\right)\left[1-\left(1-M_{h}\right)\left(1-P_{h}\right)\right]}{\left[2 P_{h}-1+2 M_{h}\left(1-P_{h}\right)\right]^{2}} .
\end{aligned}
$$

To find the optimum allocation we either maximize the precision for fixed budget or minimize the cost for fixed precision.

A linear cost function which is an adequate approximation of the actual cost incurred will be

$$
C=c_{0}+\sum_{h=1}^{L} c_{h} n_{h}
$$

where $c_{h}$ is per unit cost of measurement in the $h$ th stratum and $c_{0}$ is overhead cost.

In view of (4) to (11) the problem of finding optimum allocation is formulated as nonlinear programming problem (NLPP) as follows:

$$
\begin{array}{ll}
\text { Minimize } & V\left(\widehat{\pi}_{s}\right), \\
\text { Subject to } & \sum_{h=1}^{L} c_{h} n_{h} \leq C_{0}, \\
& 1 \leq n_{h} \leq N_{h} ; h=1,2, \ldots, L .
\end{array}
$$

The restrictions $1 \leq n_{h}$ and $n_{h} \leq N_{h}$ are placed to have the representation of every stratum in the sample and to avoid the oversampling, respectively.

\section{Fuzzy Formulation of Two-Stage Randomized Response Problem}

Generally, real-world situations involve a lot of parameters such as cost and time, whose values are assigned by the decision makers and in the conventional approach, they are required to fix an exact value to the aforementioned parameters. However decision-makers frequently do not precisely know the value of those parameters. Therefore, in such cases it is better to consider those parameters or coefficients in the decision-making problems as fuzzy numbers. The mathematical modeling of fuzzy concepts was presented by Zadeh in [21]. Therefore, the fuzzy formulation of problem (12) with fuzzy cost constraint is given by considering two cases of fuzzy numbers, that is, triangular fuzzy number (TFN) and trapezoidal fuzzy number (TrFN).

\subsection{Case 1: Nonlinear Problem with TFN. Consider}

$$
\begin{aligned}
\text { Minimize } & \sum_{h=1}^{L} \frac{W_{h}^{2}}{n_{h}}\left\{\pi_{s h}\left(1-\pi_{s h}\right)+A_{h}\right\}, \\
\text { Subject to } & \sum_{h=1}^{L}\left(c_{h}^{(1)}, c_{h}^{(2)}, c_{h}^{(3)}\right) n_{h} \leq\left(C_{0}^{(1)}, C_{0}^{(2)}, C_{0}^{(3)}\right), \\
& 1 \leq n_{h} \leq N_{h} ; h=1,2, \ldots, L,
\end{aligned}
$$

where

$$
A_{h}=\frac{\left(1-M_{h}\right)\left(1-P_{h}\right)\left[1-\left(1-M_{h}\right)\left(1-P_{h}\right)\right]}{\left[2 P_{h}-1+2 M_{h}\left(1-P_{h}\right)\right]^{2}}
$$

and $\widetilde{c}_{h}=\left(c_{h}^{(1)}, c_{h}^{(2)}, c_{h}^{(3)}\right)$ is triangular fuzzy numbers with membership function

$$
\mu_{\widetilde{c}_{h}}(x)= \begin{cases}\frac{x-c_{h}^{(1)}}{c_{h}^{(2)}-c_{h}^{(1)}}, & \text { if } c_{h}^{(1)} \leq x \leq c_{h}^{(2)}, \\ \frac{c_{h}^{(3)}-x}{c_{h}^{(3)}-c_{h}^{(2)}}, & \text { if } c_{h}^{(2)} \leq x \leq c_{h}^{(3)}, \\ 0, & \text { otherwise. }\end{cases}
$$

Similarly, the membership function for available budget can be expressed as

$$
\mu_{\widetilde{C}_{0}}(x)= \begin{cases}\frac{x-C_{0}^{(1)}}{C_{0}^{(2)}-C_{0}^{(1)}}, & \text { if } C_{0}^{(1)} \leq x \leq C_{0}^{(2)}, \\ \frac{C_{0}^{(3)}-x}{C_{0}^{(3)}-C_{0}^{(2)}}, & \text { if } C_{0}^{(2)} \leq x \leq C_{0}^{(3)}, \\ 0, & \text { otherwise. }\end{cases}
$$

\subsection{Case 2: Nonlinear Problem with TrFN. Consider}

$$
\begin{array}{ll}
\text { Minimize } & \sum_{h=1}^{L} \frac{W_{h}^{2}}{n_{h}}\left\{\pi_{s h}\left(1-\pi_{s h}\right)+A_{h}\right\} \\
\text { Subject to } & \sum_{h=1}^{L}\left(c_{h}^{(1)}, c_{h}^{(2)}, c_{h}^{(3)}, c_{h}^{(4)}\right) n_{h}
\end{array}
$$




$$
\begin{aligned}
& \leq\left(C_{0}^{(1)}, C_{0}^{(2)}, C_{0}^{(3)}, C_{0}^{(4)}\right) \\
& 1 \leq n_{h} \leq N_{h} ; h=1,2, \ldots, L
\end{aligned}
$$

where

$$
A_{h}=\frac{\left(1-M_{h}\right)\left(1-P_{h}\right)\left[1-\left(1-M_{h}\right)\left(1-P_{h}\right)\right]}{\left[2 P_{h}-1+2 M_{h}\left(1-P_{h}\right)\right]^{2}}
$$

and $\widetilde{c}_{h}=\left(c_{h}^{(1)}, c_{h}^{(2)}, c_{h}^{(3)}, c_{h}^{(4)}\right)$ is trapezoidal fuzzy numbers with membership function

$$
\mu_{\tau_{h}}(x)= \begin{cases}0, & x \leq c_{h}^{(1)}, \\ \frac{x-c_{h}^{(1)}}{c_{h}^{(2)}-c_{h}^{(1)}}, & c_{h}^{(1)} \leq x \leq c_{h}^{(2)}, \\ 1, & c_{h}^{(2)} \leq x \leq c_{h}^{(3)}, \\ \frac{c_{h}^{(4)}-x}{c_{h}^{(4)}-c_{h}^{(3)}}, & c_{h}^{(3)} \leq x \leq c_{h}^{(4)}, \\ 0, & c_{h}^{(4)} \leq x .\end{cases}
$$

Similarly, the membership function for available budget can be expressed as

$$
\mu_{\widetilde{C}_{0}}(x)= \begin{cases}0, & x \leq C_{0}^{(1)}, \\ \frac{x-C_{0}^{(1)}}{C_{0}^{(2)}-C_{0}^{(1)}}, & C_{0}^{(1)} \leq x \leq C_{0}^{(2)}, \\ 1, & C_{0}^{(2)} \leq x \leq C_{0}^{(3)}, \\ \frac{C_{0}^{(4)}-x}{C_{0}^{(4)}-C_{0}^{(3)},} & C_{0}^{(3)} \leq x \leq C_{0}^{(4)}, \\ 0, & C_{0}^{(4)} \leq x .\end{cases}
$$

\section{Lagrange Multipliers Technique}

In problem (13), after ignoring the restrictions and taking equality in cost constraint the NLPP with TFNs is solved by Lagrange multipliers technique (LMT) as follows.

The Lagrangian function can be defined as

$$
\begin{aligned}
\phi\left(n_{h}, \lambda\right) & \\
= & \sum_{h=1}^{L} \frac{W_{h}^{2}}{n_{h}}\left\{\pi_{s h}\left(1-\pi_{s h}\right)+A_{h}\right\} \\
& +\lambda\left\{\sum_{h=1}^{L}\left(c_{h}^{(1)}, c_{h}^{(2)}, c_{h}^{(3)}\right) n_{h}-\left(C_{0}^{(1)}, C_{0}^{(2)}, C_{0}^{(3)}\right)\right\} .
\end{aligned}
$$

$$
n_{h}^{*}=\frac{\left(C_{0}^{(1)}, C_{0}^{(2)}, C_{0}^{(3)}, C_{0}^{(4)}\right) W_{h} \sqrt{\left\{\pi_{s h}\left(1-\pi_{s h}\right)+A_{h}\right\} /\left(c_{h}^{(1)}, c_{h}^{(2)}, c_{h}^{(3)}, c_{h}^{(4)}\right)}}{\sum_{h=1}^{L} W_{h} \sqrt{\left\{\pi_{s h}\left(1-\pi_{s h}\right)+A_{h}\right\}\left(c_{h}^{(1)}, c_{h}^{(2)}, c_{h}^{(3)}, c_{h}^{(4)}\right)}}
$$

Differentiating (21) with respect to $n_{h}$ and $\lambda$ and equating to zero, we get the following sets of equations:

$$
\begin{aligned}
\frac{\partial \phi}{\partial n_{h}} & =-\frac{W_{h}^{2}}{n_{h}^{2}}\left\{\pi_{s h}\left(1-\pi_{s h}\right)+A_{h}\right\}+\lambda\left(c_{h}^{(1)}, c_{h}^{(2)}, c_{h}^{(3)}\right) \\
& =0
\end{aligned}
$$

Also,

$$
\frac{\partial \phi}{\partial \lambda}=\left\{\sum_{h=1}^{L}\left(c_{h}^{(1)}, c_{h}^{(2)}, c_{h}^{(3)}\right) n_{h}-\left(C_{0}^{(1)}, C_{0}^{(2)}, C_{0}^{(3)}\right)\right\}=0
$$

which gives

$$
\begin{aligned}
& \frac{1}{\sqrt{\lambda}} \\
& =\frac{\left(C_{0}^{(1)}, C_{0}^{(2)}, C_{0}^{(3)}\right)}{\sum_{h=1}^{L} W_{h} \sqrt{\left\{\pi_{s h}\left(1-\pi_{s h}\right)+A_{h}\right\}\left(c_{h}^{(1)}, c_{h}^{(2)}, c_{h}^{(3)}\right)}} .
\end{aligned}
$$

In similar manner, the optimum allocation of NLPP (17) with trapezoidal fuzzy numbers can be obtain as

Now using (23) and (26), we obtain

$$
\begin{aligned}
& n_{h}^{*} \\
& =\frac{\left(C_{0}^{(1)}, C_{0}^{(2)}, C_{0}^{(3)}\right) W_{h} \sqrt{\left\{\pi_{s h}\left(1-\pi_{s h}\right)+A_{h}\right\} /\left(c_{h}^{(1)}, c_{h}^{(2)}, c_{h}^{(3)}\right)}}{\sum_{h=1}^{L} W_{h} \sqrt{\left\{\pi_{s h}\left(1-\pi_{s h}\right)+A_{h}\right\}\left(c_{h}^{(1)}, c_{h}^{(2)}, c_{h}^{(3)}\right)}} .
\end{aligned}
$$


The allocations obtained in (27) and (28) are fuzzy in nature, so we have to convert fuzzy allocations into a crisp allocation by $\alpha$-cut method at a prescribed value of $\alpha$.

\section{Procedure for the Conversion of Fuzzy Numbers}

To convert the fuzzy allocation into crisp allocation $\alpha$-cut method is used as follows.

Let $\widetilde{A}=(p, q, r)$ be a TFN. An $\alpha$-cut for $\widetilde{A}, \widetilde{A}_{\alpha}$ is computed as

$$
\alpha=\frac{x-p}{q-p} \Longrightarrow \widetilde{A}_{\alpha}^{L}=x=(q-p) \alpha+p
$$

$$
\alpha=\frac{r-x}{r-q} \Longrightarrow \widetilde{A}_{\alpha}^{U}=x=r-(r-q) \alpha,
$$

where $\widetilde{A}_{\alpha}=\left[\widetilde{A}_{\alpha}^{L}, \widetilde{A}_{\alpha}^{U}\right]$ is the corresponding $\alpha$-cut (see Figure 1).

The allocation obtained in (27) is in the form of triangular fuzzy number; therefore by using (29) the equivalent crisp allocation is given by

$$
n_{h}^{*}=\frac{\left(C_{0}^{(3)}-\left(C_{0}^{(3)}-C_{0}^{(2)}\right)\right) W_{h} \sqrt{\left\{\pi_{s h}\left(1-\pi_{s h}\right)+A_{h}\right\} /\left(\left(c_{h}^{(2)}-c_{h}^{(1)}\right)+c_{h}^{(1)}\right)}}{\sum_{h=1}^{L} W_{h} \sqrt{\left\{\pi_{s h}\left(1-\pi_{s h}\right)+A_{h}\right\}\left(\left(c_{h}^{(2)}-c_{h}^{(1)}\right)+c_{h}^{(1)}\right)}} .
$$

Similarly, if $\widetilde{A}=(p, q, r, s)$ is a $\operatorname{TrFN}$, then the $\alpha$-cut for $\widetilde{A}, \widetilde{A}_{\alpha}$ is computed as

$$
\begin{aligned}
& \alpha=\frac{x-p}{q-p} \Longrightarrow \widetilde{A}_{\alpha}^{L}=x=(q-p) \alpha+p, \\
& \alpha=\frac{s-x}{s-r} \Longrightarrow \widetilde{A}_{\alpha}^{U}=x=s-(s-r) \alpha,
\end{aligned}
$$

where $\widetilde{A}_{\alpha}=\left[\widetilde{A}_{\alpha}^{L}, \widetilde{A}_{\alpha}^{U}\right]$ is the corresponding $\alpha$-cut (see Figure 2).

In similar manner, the crisp allocation corresponding to (28) is given by

$$
n_{h}^{*}=\frac{\left(C_{0}^{(4)}-\left(C_{0}^{(4)}-C_{0}^{(3)}\right)\right) W_{h} \sqrt{\left\{\pi_{s h}\left(1-\pi_{s h}\right)+A_{h}\right\} /\left(\left(c_{h}^{(2)}-c_{h}^{(1)}\right)+c_{h}^{(1)}\right)}}{\sum_{h=1}^{L} W_{h} \sqrt{\left\{\pi_{s h}\left(1-\pi_{s h}\right)+A_{h}\right\}\left(\left(c_{h}^{(2)}-c_{h}^{(1)}\right)+c_{h}^{(1)}\right)}} .
$$

The allocations obtained by (30) and (32) provide the solution to NLPP (13) and (17) if it satisfies the restriction $1 \leq n_{h} \leq N_{h}$; $h=1,2, \ldots, L$. The allocations obtained in (30) and (32) may not be integer allocations, so to get integer allocations, round off the allocations to the nearest integer values. After rounding off we have to be careful in rechecking that the round-off values satisfy the cost constraint.

\section{Some Other Allocation Techniques}

7.1. Equal Allocation. In this method, the total sample size $n$ is divided equally among all the strata, that is, for the $h$ th stratum

$$
n_{h}=\frac{n}{L}
$$

where $n$ can be obtained from the cost constraint equation as follows:

$$
\sum_{h=1}^{L}\left(\left(c_{h}^{(2)}-c_{h}^{(1)}\right)+c_{h}^{(1)}\right) n_{h}=\left(C_{0}^{(4)}-\left(C_{0}^{(4)}-C_{0}^{(3)}\right)\right)
$$

$$
\begin{gathered}
n_{h} \propto W_{h} \\
\text { or } n_{h}=n W_{h} .
\end{gathered}
$$

Now substituting the value of $n_{h}$ in (34), we get

$$
n=\frac{N\left(C_{0}^{(4)}-\left(C_{0}^{(4)}-C_{0}^{(3)}\right)\right)}{\sum_{h=1}^{L}\left(\left(c_{h}^{(2)}-c_{h}^{(1)}\right)+c_{h}^{(1)}\right) N_{h}} .
$$

7.2. Proportional Allocation. This allocation was originally proposed by Bowley [22]. This procedure of allocation is very common in practice because of its simplicity. When no other information except $N_{h}$, the total number of units in the $h$ th stratum, is available, the allocation of a given sample of size $n$ to different strata is done in proportion to their sizes, that is, in the $h$ th stratum

$$
n_{h}=n \frac{N_{h}}{N}
$$




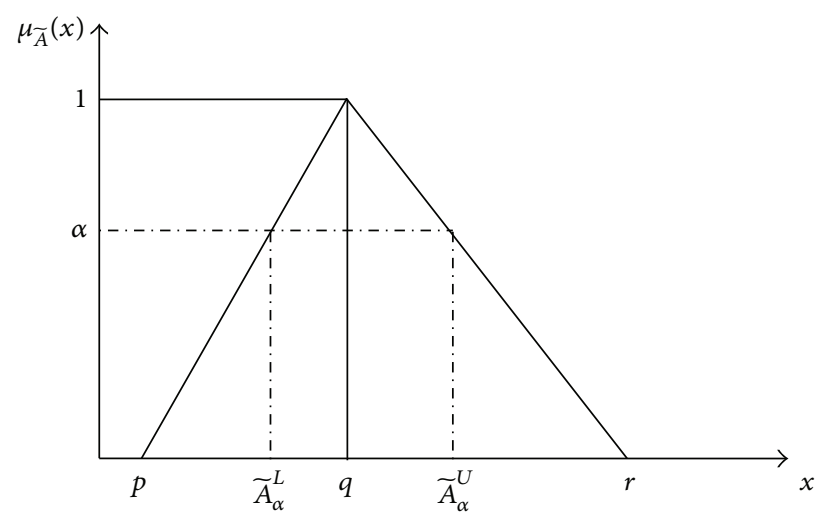

FIGURE 1: Triangular fuzzy number with an $\alpha$-cut.

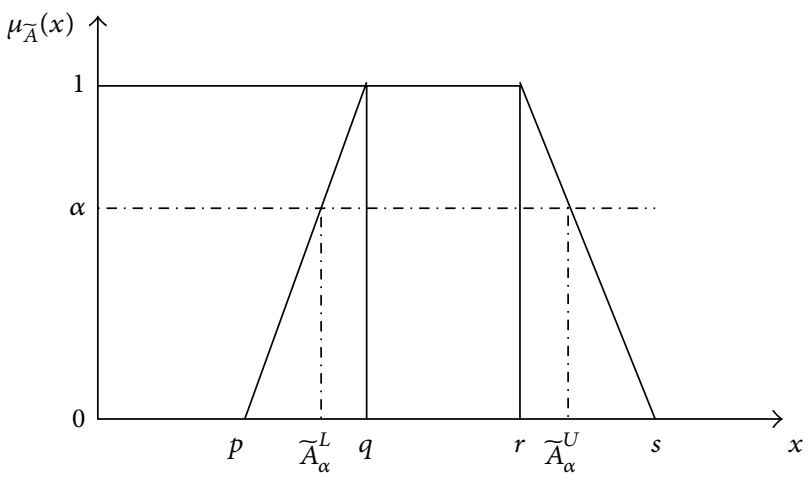

FIgURE 2: Trapezoidal fuzzy number with an $\alpha$-cut.

7.3. Ghufran's Allocation. Ghufran et al. [17] formulate a crisp NLPP

$$
\begin{array}{ll}
\text { Minimize } & \sum_{h=1}^{L} \frac{W_{h}^{2}}{n_{h}}\left\{\pi_{s h}\left(1-\pi_{s h}\right)+A_{h}\right\}, \\
\text { Subject to } & \sum_{h=1}^{L} c_{h} n_{h} \leq C_{0}, \\
& 1 \leq n_{h} \leq N_{h} ; h=1,2, \ldots, L
\end{array}
$$

and obtained an optimum allocation using LMT as

$$
n_{h}^{*}=\frac{C_{0} W_{h} \sqrt{\left\{\pi_{s h}\left(1-\pi_{s h}\right)+A_{h}\right\} / c_{h}}}{\sum_{h=1}^{L} W_{h} \sqrt{\left\{\pi_{s h}\left(1-\pi_{s h}\right)+A_{h}\right\} c_{h}}} .
$$

\section{Numerical Illustration}

A hypothetical example is given to illustrate the computational details of the proposed problem. Let us suppose the population size is 1000 with total available budget of the survey as TFNs and TrFNs are $(3500,4000,4800)$ and $(3500,4000,4400,4600)$ units, respectively. The other required relevant information is given in Table 1 . By using the values of Table 1 , we compute the values of $A_{h}$ which is given in Table 2.

After substituting all the values from Tables 1 and 2 in (13), the required FNLPP is given as

$$
\begin{array}{ll}
\text { Minimize } & \frac{0.03098772}{n_{1}}+\frac{0.20718425}{n_{2}} \\
\text { Subject to } & (1,2,4) n_{1}+(18,20,24) n_{2} \\
& \leq(3500,4000,4800), \\
& 1 \leq n_{1} \leq 300 ; 1 \leq n_{2} \leq 700 .
\end{array}
$$

The required optimum allocations for problem (13) obtained by substituting the values from Tables 1 and 2 in (30) at $\alpha=$ 0.5 will be

$$
\begin{aligned}
& n_{1}=\frac{(4800-800 \alpha) \times 0.3 \sqrt{\{0.4(1-0.4)+0.104308\} /(\alpha+1)}}{0.3 \sqrt{\{0.4(1-0.4)+0.104308\}(\alpha+1)}+0.3 \sqrt{\{0.6(1-0.6)+0.182825\}(2 \alpha+18)}}=287.51 \simeq 288, \\
& n_{2}=\frac{(4800-800 \alpha) \times 0.7 \sqrt{\{0.6(1-0.6)+0.182825\} /(2 \alpha+18)}}{0.3 \sqrt{\{0.4(1-0.4)+0.104308\}(\alpha+1)}+0.3 \sqrt{\{0.6(1-0.6)+0.182825\}(2 \alpha+18)}}=208.88 \simeq 209 .
\end{aligned}
$$

In similar manner, optimum allocations for problem (17) obtained by substituting the values from Tables 1 and 2 in (32) at $\alpha=0.5$ will be

$$
n_{1}=\frac{3750 \times 0.3 \sqrt{\{0.4(1-0.4)+0.104308\} /(\alpha+1)}}{0.3 \sqrt{\{0.4(1-0.4)+0.104308\}(\alpha+1)}+0.3 \sqrt{\{0.6(1-0.6)+0.182825\}(2 \alpha+18)}}=280.97 \simeq 281
$$


TABLE 1: Data for two strata.

\begin{tabular}{lllllll}
\hline Stratum $h$ & $W_{h}$ & $\pi_{s h}$ & $M_{h}$ & $P_{h}$ & $\left(c_{h}^{(1)}, c_{h}^{(2)}, c_{h}^{(3)}\right)$ & $\left(C_{0}^{(1)}, C_{0}^{(2)}, C_{0}^{(3)}\right)$ \\
\hline 1 & 0.3 & 0.4 & 0.8 & 0.6 & $(1,2,4)$ & $(1,2,4,7)$ \\
2 & 0.7 & 0.6 & 0.6 & 0.7 & $(18,20,24)$ & $(18,20,24,26)$ \\
\hline
\end{tabular}

TABle 2: Computation for $A_{h}$.

\begin{tabular}{lccccccccccc}
\hline$(1)$ & $(2)$ & $(3)$ & $(4)$ & $(5)$ & $(6)$ & $(7)$ & $(8)$ & $(9)$ & $(10)$ & $(11)$ & $(12)$ \\
\hline Stratum $h$ & $M_{h}$ & $P_{h}$ & $\left(1-M_{h}\right)$ & $\left(1-P_{h}\right)$ & $(4) \times(5)$ & $1-(6)$ & $\left(2 P_{h}-1\right)$ & $2 M_{h} \times(5)$ & {$[(8)+(9)]^{2}$} & $(6) \times(7)$ & $A_{h}=(11) /(10)$ \\
\hline 1 & 0.8 & 0.6 & 0.2 & 0.4 & 0.08 & 0.92 & 0.2 & 0.64 & 0.7056 & 0.0736 & 0.104308 \\
2 & 0.6 & 0.7 & 0.4 & 0.3 & 0.12 & 0.88 & 0.4 & 0.36 & 0.5776 & 0.1056 & 0.182825 \\
\hline
\end{tabular}

TABLE 3: Optimum allocations.

\begin{tabular}{lcccc}
\hline & & Allocations & $n_{2}$ & Variance \\
\hline \multirow{2}{*}{ LMT (optimum allocation) } & Case of TFN & $n_{1}$ & 209 & $\mathbf{0 . 0 0 1 0 9 8 9 0 8}$ \\
& Case of TrFN & 288 & 204 & $\mathbf{0 . 0 0 1 1 2 5 8 8 6}$ \\
\hline \multirow{2}{*}{ Equal allocation } & Case of TFN & 281 & 160 & 0.001488575 \\
& Case of TrFN & 160 & 156.5 & 0.001521866 \\
\hline Proportional allocation & & 41 & 95 & 0.002936685 \\
Ghufran's allocation & & 86 & 157 & 0.00167996 \\
\hline
\end{tabular}

$$
n_{2}=\frac{(4400-200 \alpha) \times 0.7 \sqrt{\{0.6(1-0.6)+0.182825\} /(2 \alpha+18)}}{0.3 \sqrt{\{0.4(1-0.4)+0.104308\}(\alpha+1)}+0.3 \sqrt{\{0.6(1-0.6)+0.182825\}(2 \alpha+18)}}=204.13 \simeq 204
$$

By using $\alpha$-cut and LMT, the optimum allocation after rounding-off is obtained and summarized in Table 3 with the equal allocation, proportional allocation, and Ghufran's allocation.

\section{Conclusion}

The optimum allocation problem in two-stage stratified warner's randomized response model with fuzzy costs is formulated as a problem of fuzzy nonlinear programming problem. The problem is then solved by using Lagrange multipliers technique for obtaining optimum allocation. The optimum allocation obtained in the form of fuzzy numbers is converted into an equivalent crisp number by using $\alpha$-cut method at a prescribed value of $\alpha$.

On comparing the result of LMT with the result of equal allocation, proportional allocation, and Ghufran's allocation, it is seen that LMT gives the best allocation. But it is not necessary that optimum allocation obtained by Lagrange multipliers technique always gives the feasible or optimal solution (proved by [17]) and also for practical purposes we need integer sample sizes. Therefore, in future instead of rounding off the continuous solution, we can obtain integer solution by Dakin's Method [23] or formulating the problem as fuzzy integer nonlinear programming problem and obtain the integer solution by LINGO software.

\section{Conflict of Interests}

The authors declare that there is no conflict of interests regarding the publication of this paper.

\section{Acknowledgment}

The third author is thankful to University Grant Commission for providing financial assistance under the UGC Start-up Grant no. F.30-90/2015 (BSR), Delhi, India, to carry out this research work.

\section{References}

[1] S. L. Warner, "Randomized response: a survey technique for eliminating evasive answer bias," Journal of the American Statistical Association, vol. 60, no. 309, pp. 63-69, 1965.

[2] N. S. Mangat and R. Singh, "An alternative randomized response procedure," Biometrika, vol. 77, no. 2, pp. 439-442, 1990. 
[3] T. C. Chua and A. K. Tsui, "Procuring honest responses indirectly," Journal of Statistical Planning and Inference, vol. 90, no. 1, pp. 107-116, 2000.

[4] V. R. Padmawar and K. Vijayan, "Randomized response revisited," Journal of Statistical Planning and Inference, vol. 90, no. 2, pp. 293-304, 2000.

[5] H.-J. Chang and K.-C. Huang, "Estimation of proportion and sensitivity of a qualitative character," Metrika, vol. 53, no. 3, pp. 269-280, 2001.

[6] A. Chaudhuri, "Using randomized response from a complex survey to estimate a sensitive proportion in a dichotomous finite population," Journal of Statistical Planning and Inference, vol. 94, no. 1, pp. 37-42, 2001.

[7] J.-M. Kim and W. D. Warde, "A stratified Warner's randomized response model," Journal of Statistical Planning and Inference, vol. 120, no. 1-2, pp. 155-165, 2004.

[8] R. E. Bellman and L. A. Zadeh, "Decision-making in a fuzzy environment," Management Science, vol. 17, no. 4, pp. B-141-B$164,1970$.

[9] H. Tanaka and K. Asai, "Fuzzy linear programming problem with fuzzy numbers," Fuzzy Sets and Systems, vol. 13, no. 1, pp. 1-10, 1984.

[10] G. S. Mahapatra and T. K. Roy, "Fuzzy multi-objective mathematical programming on reliability optimization model," Applied Mathematics and Computation, vol. 174, no. 1, pp. 643659, 2006.

[11] S. Pramanik and T. K. Roy, "Multiobjective transportation model with fuzzy parameters: priority based fuzzy goal programming approach," Journal of Transportation Systems Engineering \& Information Technology, vol. 8, no. 3, pp. 40-48, 2008.

[12] S. Abbasbandy and T. Hajjari, "A new approach for ranking of trapezoidal fuzzy numbers," Computers and Mathematics with Applications, vol. 57, no. 3, pp. 413-419, 2009.

[13] A. Kaur and A. Kumar, "A new approach for solving fuzzy transportation problems using generalized trapezoidal fuzzy numbers," Applied Soft Computing, vol. 12, no. 3, pp. 1201-1213, 2012.

[14] A. Ebrahimnejad, "A simplified new approach for solving fuzzy transportation problems with generalized trapezoidal fuzzy numbers," Applied Soft Computing, vol. 19, pp. 171-176, 2014.

[15] N. Sen, L. Sahoo, and A. K. Bhunia, "An application of integer linear programming problem in tea industry of barak valley of Assam, India under crisp and fuzzy environments," Journal of Information and Computing Science, vol. 9, no. 2, pp. 132-140, 2014.

[16] N. Gupta and A. Bari, "Multi-choice goal programming with trapezoidal fuzzy numbers," International Journal of Operations Research, vol. 11, no. 3, pp. 82-90, 2014.

[17] S. Ghufran, S. Khowaja, and M. J. Ahsan, "Optimum allocation in two-stage stratified randomized response model," Journal of Mathematical Modelling and Algorithms, vol. 12, no. 4, pp. 383392, 2013.

[18] C. R. Bector and S. Chandra, Fuzzy Mathematical Programming and Fuzzy Matrix Games, vol. 169, Springer, Berlin, Germany, 2005.

[19] R. Hassanzadeh, I. Mahdavi, N. M. Amiri, and A. Tajdin, "An $\alpha$-cut approach for fuzzy product and its use in computing solutions of fully fuzzy linear systems," in Proceedings of the International Conference on Industrial Engineering and Operations Management (IEOM '12), Istanbul, Turkey, July 2012.
[20] S. Aggarwal and U. Sharma, "Fully fuzzy multi-choice multiobjective linear programming solution via deviation degree," International Journal of Pure and Applied Sciences and Technology, vol. 19, no. 1, pp. 49-64, 2013.

[21] L. A. Zadeh, "Fuzzy sets," Information and Computation, vol. 8, no. 3, pp. 338-353, 1965.

[22] A. L. Bowley, Measurements of the Precision Attained in Sampling, vol. 22, no. 1 of Bulletin of the International Statistical Institute, Cambridge University Press, Cambridge, UK, 1926.

[23] R. J. Dakin, "A tree-search algorithm for mixed integer programming problems," The Computer Journal, vol. 8, no. 3, pp. 250-255, 1965. 

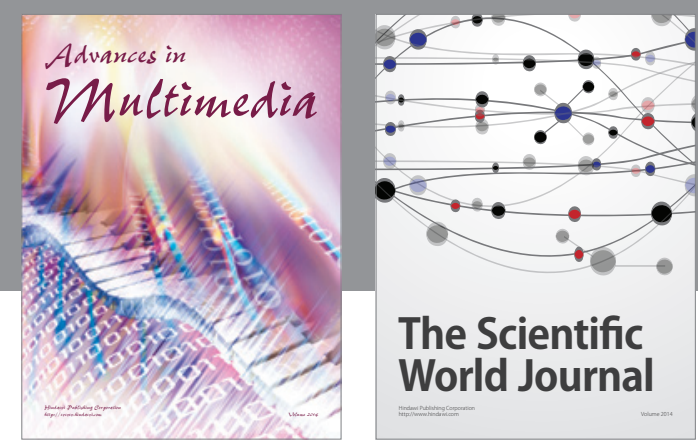

The Scientific World Journal
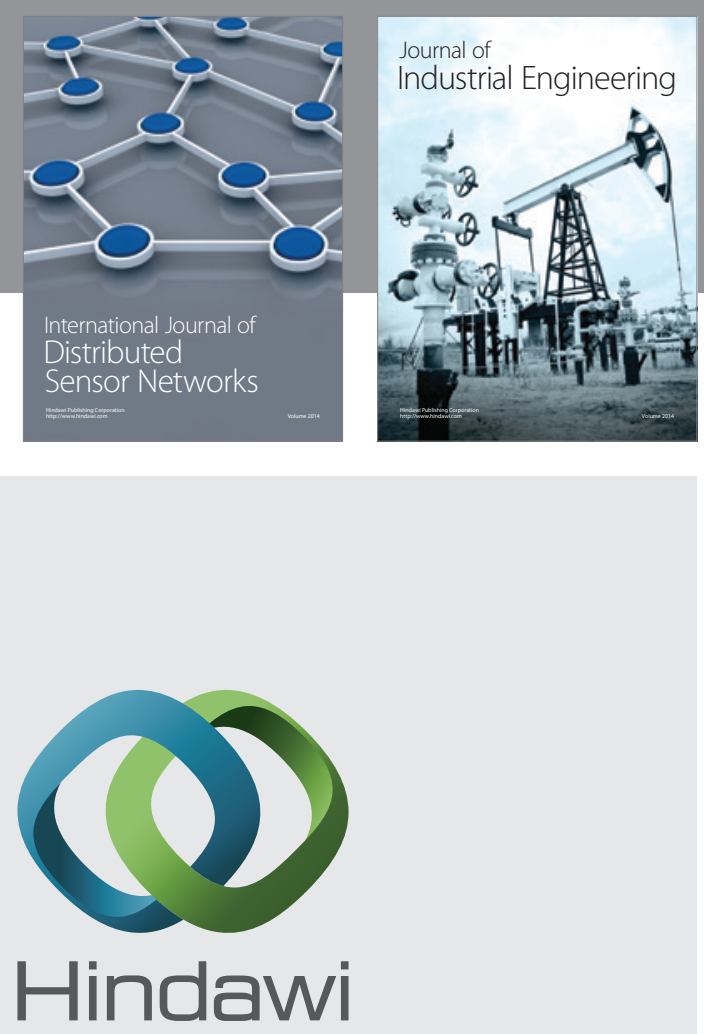

Submit your manuscripts at

http://www.hindawi.com

\section{Computer Networks} and Communications
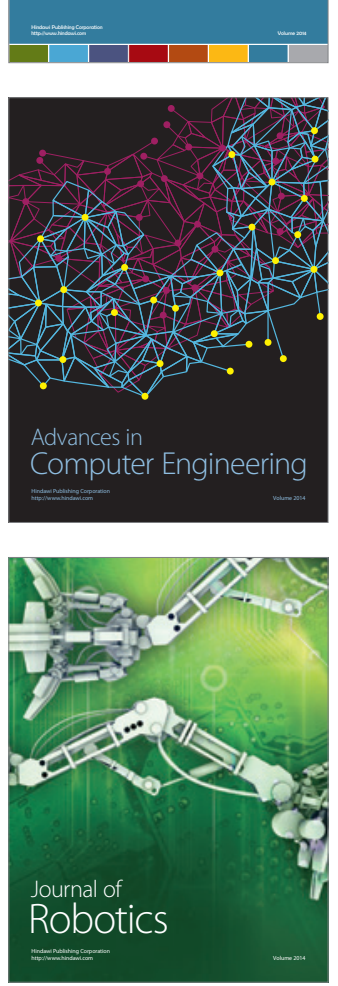
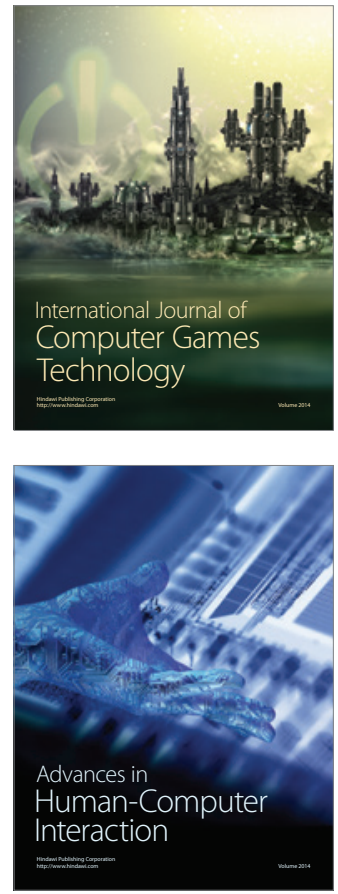
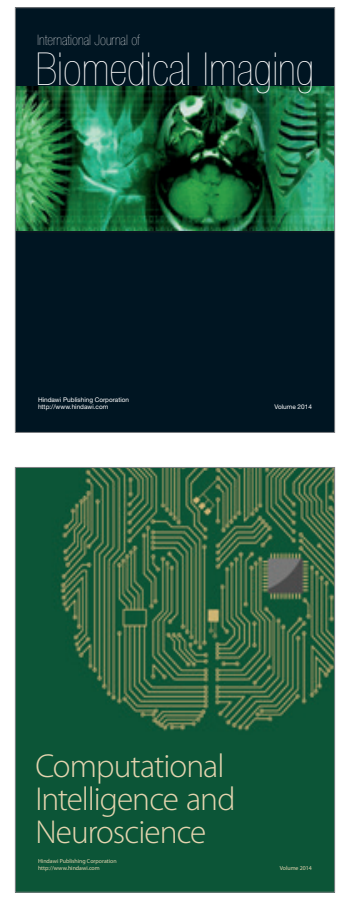
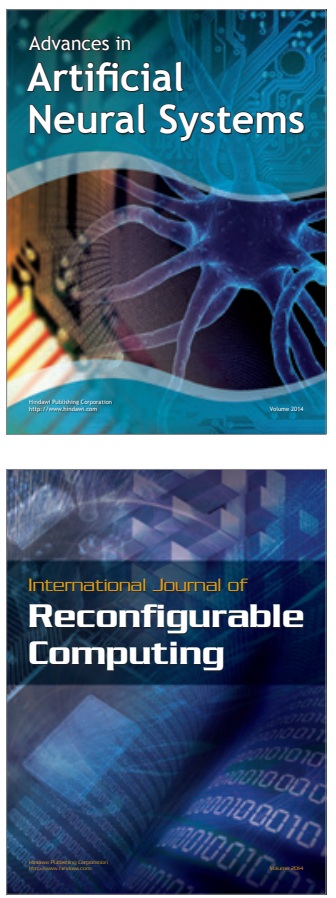
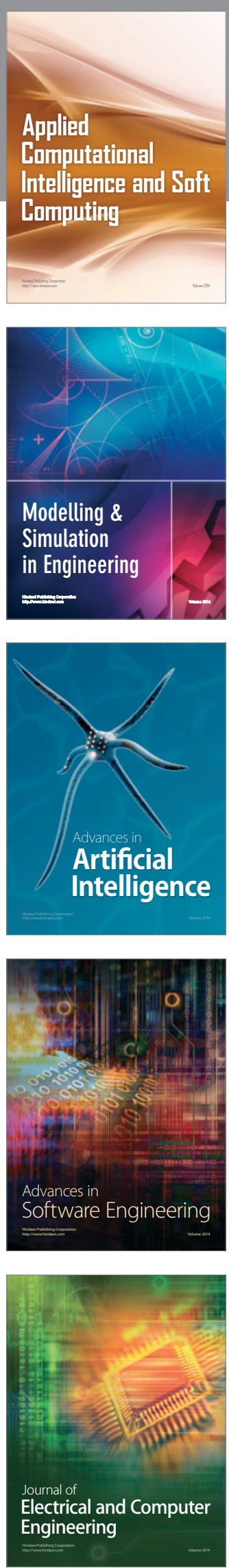